\title{
The effects of Bifidobacterium animalis ssp. lactis B94 on gastrointestinal wellness in adults with Prader-Willi syndrome: study protocol for a randomized controlled trial
}

\author{
Zainab Alyousif ${ }^{1}$, Jennifer L. Miller ${ }^{2}$, Mariana Y. Sandoval ${ }^{3}$, Chad W. MacPherson ${ }^{3}$, Varuni Nagulesapillai ${ }^{3}$ \\ and Wendy J. Dahl ${ }^{1 *}$ (D)
}

\begin{abstract}
Background: Constipation is a frequent problem in adults with Prader-Willi syndrome. Certain probiotics have been shown to improve transit and gastrointestinal symptoms of adults with functional constipation. The aim of this study is to determine the effect of daily consumption of Bifidobacterium animalis ssp. lactis B94 (B. lactis B94) on stool frequency, stool form, and gastrointestinal symptoms in adults with Prader-Willi syndrome.

Methods: Adults with Prader-Willi syndrome (18-75 years old, $n=36)$ will be recruited and enrolled in a 20-week, randomized, double-blind, placebo-controlled, crossover study. Study subjects will be randomized to B. lactis B94 or placebo each for a 4-week period, preceded by a 4-week baseline and followed by 4-week washouts. Subjects will complete daily records of stool frequency and stool form (a proxy of transit time). Dietary intake data also will be collected. Stools, one in each period, will be collected for exploratory microbiota analyses.

Discussion: To our knowledge, this is the first randomized controlled trial evaluating the effectiveness of $B$. lactis in adults with Prader-Willi syndrome. The results of this study will provide evidence of efficacy for future clinical trials in patient populations with constipation.
\end{abstract}

Trial registration: ClinicalTrials.gov (NCT03277157). Registered on 08 September 2017.

Keywords: Bifidobacterium, Prader-Willi syndrome, constipation, gastrointestinal function, microbiome

\section{Background}

Prader-Willi syndrome (PWS) is a genetic disorder due to the non-expression of specific genes from the chromosome 15q11.2-q13 region [1]. The lack of imprinted genes occurs by three mechanisms, namely deletion of the 15q11.2-q13 region (found in $65-75 \%$ of individuals), maternal uniparental disomy (found in $20-30 \%$ of individuals), and an imprinting defect when the genomic region that manages the imprinting process is defective $(1-3 \%$ of individuals) [1]. The estimated prevalence of PWS is 1/ 10,000 to $1 / 30,000$ people [1]. At an early age, infants have severe hypotonia and feeding difficulties followed by

\footnotetext{
* Correspondence: wdahl@ufl.edu

${ }^{1}$ Food Science and Human Nutrition Department, UF/IFAS, University of Florida, 359 FSHN Building Newell Drive, Gainesville, FL 32611, USA Full list of author information is available at the end of the article
}

weight gain and subsequent hyperphagia during childhood [1]. Due to altered body composition and depressed basal metabolic rate in adults with PWS [2], lifelong energy restriction is required to circumvent the development of morbid obesity [3].

Constipation is a common problem for individuals with PWS. A study of adults with PWS showed that $40 \%$ of these individuals have constipation symptoms, including having less than three defecations per week, sensation of anorectal obstruction, straining during defecation, and having hard stools [4]. Although adequate dietary fiber may be achievable in children with PWS [5], this may be a challenge in adults with PWS, particularly given their low energy needs and, thus, limited food choices. Certain probiotics, "live microorganisms that when administered in adequate amounts

(c) The Author(s). 2018 Open Access This article is distributed under the terms of the Creative Commons Attribution 4.0 International License (http://creativecommons.org/licenses/by/4.0/), which permits unrestricted use, distribution, and reproduction in any medium, provided you give appropriate credit to the original author(s) and the source, provide a link to the Creative Commons license, and indicate if changes were made. The Creative Commons Public Domain Dedication waiver (http://creativecommons.org/publicdomain/zero/1.0/) applies to the data made available in this article, unless otherwise stated. 
confer a health benefit to the host" [6], have been shown to improve constipation symptoms in otherwise healthy adults. Probiotics have been shown to significantly decrease transit time, increase stool frequency, and improve stool consistency, particularly Bifidobacterium lactis strains [7]. This study will build on previous literature reporting on the efficacy of probiotics in adults with constipation and will examine the effect of Bifidobacterium animalis ssp. lactis B94 (B. lactis B94) in adults with PWS. The aim of the study is to determine the effect of B. lactis B94 on stool frequency, stool form (a proxy for transit time), gastrointestinal (GI) symptoms, and fecal microbiota in adults with PWS. It is hypothesized that B. lactis B94 will increase stool frequency, decrease the percentage of slow transit stools, and improve GI symptoms. The primary outcome of the study is weekly stool frequency (difference between treatments), whereas secondary outcomes are weekly stool frequency (percentage change from baseline), stool form (percentage change in slow transit Bristol Stool Form Scale (BSFS) 1 and 2 [8] from baseline and between treatments), GI symptoms, and compliance (recorded intake of the provided and remaining supplements).

\section{Methods}

\section{Design}

A 20-week randomized, double-blinded, placebo-controlled crossover study will be carried out (Fig. 1). Adults with genetically confirmed PWS will be recruited. Subjects will complete a 4-week baseline period during which data on daily stool frequency and stool form will be collected. Dietary intake data (food record) will be obtained during the baseline period and subjects will collect a single stool. Subjects will be randomized on or about day 29 and will consume one capsule per day of B. lactis B94 or placebo for 4 weeks, followed by a 4-week washout, 4 weeks on the alternative treatment, and a second 4-week washout.
During the intervention and washout periods, subjects will continue a daily record of stool frequency, stool form, and compliance. In addition, subjects will complete the Gastrointestinal Symptom Rating Scale (GSRS) $[9,10]$ during weeks 4, 8, 12, 16, and 20. During these same weeks, dietary intake data (food record) and single stools will be collected per period. Subject demographics, height, and weight will be taken at baseline, and body weight during weeks $8,12,16$, and 20. Figure 2 provides the schedule of enrollment, interventions, and assessments to be completed. This paper follows the Standard Protocol Items: Recommendations for Interventional trials (SPIRIT) (Additional file 1) and the World Health Organization Trial Registration Data Set (Additional file 2).

\section{Randomization and blinding}

A computer-generated random number sequence will be used for allocation. Randomization will be by sealed envelope method, prepared by an individual not affiliated with the study. No blocking or stratification will be performed. The principal investigator (PI) will conduct the randomization and distribution of the investigational product (IP)/placebo. Researchers, trial participants, data analysts, and all staff members at the study sites will remain blinded for the duration of the study and until the statistical analyses are completed.

\section{Participants}

Adults with PWS, residing in residential programs and in the community at large in the state of Florida, United States, will be recruited for this study $(n=36)$. Potential subjects will be identified and contacted by their physician. The study coordinator will then meet with interested individuals, provide them with a full description of the study, and enroll those eligible. Potential subjects will be included if they have a genetically confirmed

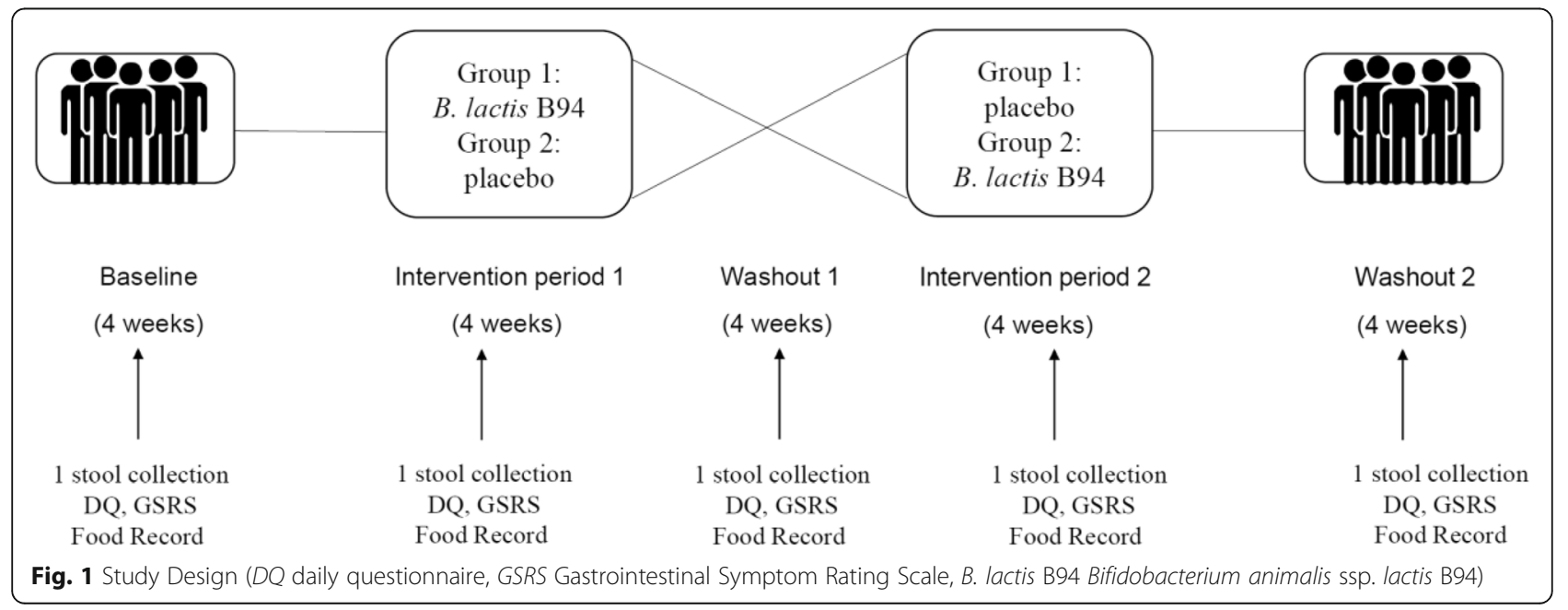




\begin{tabular}{|c|c|c|c|c|c|c|}
\hline & \multicolumn{6}{|c|}{ STUDY PERIOD } \\
\hline & Enrolment & Baseline & Intervention 1 & Washout 1 & Intervention 2 & Washout 2 \\
\hline TIMEPOINT** & $-T_{1}$ & $T_{0}$ & $T_{1}$ & $T_{2}$ & $T_{3}$ & $T_{4}$ \\
\hline \multicolumn{7}{|l|}{ ENROLMENT: } \\
\hline \multirow{2}{*}{$\begin{array}{c}\text { Eligibility screen } \\
\text { Informed consent }\end{array}$} & $x$ & & & & & \\
\hline & $x$ & & & & & \\
\hline \multirow[t]{2}{*}{ Demographic } & & $x$ & & & & \\
\hline & & & $x$ & & & \\
\hline \multicolumn{7}{|l|}{ INTERVENTIONS: } \\
\hline \multirow{2}{*}{$\begin{array}{r}\text { B. lactis B94 } \\
\text { Placebo }\end{array}$} & & & $x$ & & $x$ & \\
\hline & & & $x$ & & $x$ & \\
\hline \multicolumn{7}{|l|}{ ASSESSMENTS: } \\
\hline Height & & $x$ & & & & \\
\hline \multirow[t]{2}{*}{ Weight } & & $x$ & $x$ & $x$ & $x$ & $x$ \\
\hline & & $x$ & $x$ & $x$ & $x$ & $x$ \\
\hline Daily Questionnaire & & $x$ & $x$ & $x$ & $x$ & $x$ \\
\hline \multirow{2}{*}{$\begin{array}{r}\text { Bristol Stool Form } \\
\text { GSRS }^{*}\end{array}$} & & $x$ & $x$ & $x$ & $x$ & $x$ \\
\hline & & $x$ & $x$ & $x$ & $x$ & $x$ \\
\hline Food Record & & $x$ & $x$ & $x$ & $x$ & $x$ \\
\hline
\end{tabular}

Fig. 2 Schedule of enrollment, interventions, and assessments as per Standard Protocol Items: Recommendations for Interventional trials (SPIRIT)

diagnosis of PWS and are 18-75 years of age, willing to have height and weight measured and to provide demographic information (e.g., age, race, sex), willing to consume B. lactis B94 and placebo each for a 4-week period, willing to complete a daily record of stool number and form throughout the 20-week period, willing to complete the GSRS monthly, and willing to provide information about their dietary intake every 4 weeks. Potential subjects will be excluded if they have a milk protein allergy, are currently taking medications for diarrhea or probiotics supplements and do not want to discontinue prior to the start of the baseline period (i.e., those that discontinue will be included), and were previously or are currently being treated for GI diseases, including gastric ulcers, Crohn's disease, celiac disease, ulcerative colitis, or GI cancer. Subjects will receive their usual clinical care throughout the study.

\section{Intervention}

Subjects will be randomized to consume either B. lactis B94 or placebo for a 4-week period. A 4-week supply of the $B$. lactis B94 or placebo will be given during the study visit at the start of the intervention periods. Capsules will be stored at refrigerator temperature. The IP will be B. lactis B94 at a dose of 15 billion colony forming units (CFU) in Veggie capsule \#1 made with potato starch and magnesium stearate, and the placebo will contain potato starch and magnesium stearate
(Lallemand Health Solutions Inc., Mirabel, Canada). Prior to delivery to the study site, the IP and placebo will be coded by an unblinded employee of the manufacturer. The IP and placebo will be coded with five codes each, so in the event of an adverse event that requires unblinding of the effected subject, information about most remaining subjects will remain blinded. The IP and placebo capsules will be stored in a locked refrigerator at the research site with access limited to the PI and study coordinator.

\section{Compliance}

Study subjects will be asked to return unused capsules at the end of each intervention period to document compliance. Compliant subjects will be defined as more than $80 \%$ of supplement intake.

Recovery of B. lactis B94 from fecal samples will also be used to assess compliance. Study coordinators will be in frequent contact with the study participants throughout the study to promote compliance to the protocol.

\section{Withdrawal criteria}

Subjects will be free to withdraw their consent and to stop participating in the study at any time. Subjects may be withdrawn from the study by the PI for noncompliance (e.g., do not consume the study capsules). Consent forms, given to all subjects, will specify the contact person in the case of adverse events occurring during the study. In a circumstance that a professional 
intervention is necessary due to an adverse event, the PI will contact the study's affiliated physician and appropriate authority or organization pertinent to the circumstance of the event. If deemed necessary, the manufacturer of the capsules will be contacted to unblind the subject (one of 10 codes). As there are no known risks for consuming the probiotic, B. lactis B94, no negative side effects or adverse events are expected and, thus, no data monitoring committee is needed.

\section{Outcomes}

\section{Stool form}

Stool form will be assessed using the BSFS, a simple tool for estimating intestinal transit time [8]. The BSFS classifies stools into seven categories, including type 1, separate hard lumps, like nuts; type 2, sausage-shaped, but lumpy; type 3 , like a sausage but with cracks on the surface; type 4, like a sausage or snake, smooth and soft; type 5, soft blobs with clear-cut edges; type 6, fluffy pieces with ragged edges, a mushy stool; type 7 , watery, no solid pieces [8]. These types are categorized into slow transit (types 1 and 2), normal transit (types 3-5), and fast transit (types 6 and 7).

\section{Gl symptoms}

GI symptoms will be assessed using the GSRS, a 7-point scale that evaluates symptoms of GI disorders, where 1 represents no discomfort at all and 7 represents very severe discomfort over the past week $[9,10]$. Symptoms of the GSRS are grouped into five GI syndromes, which are abdominal pain, reflux syndrome, diarrhea syndrome, indigestion syndrome, and constipation syndrome.

\section{Microbiota analysis}

Subjects will be asked to collect one stool each during the baseline, intervention 1 , washout 1 , intervention 2 , and washout 2 periods. Subjects will be provided with Fisherbrand ${ }^{\circ}$ commode collection kits for stool collection. Samples will be processed and appropriately stored in the lab within $6 \mathrm{~h}$ after defecation. Stool samples will be analyzed for changes in microbiota. Changes in the concentration of Lactobacilli and Bifidobacteria genera as well as Bifidobacterium animalis sp. lactis will be measured in fecal samples by real-time PCR (qPCR).

Total DNA will be extracted from homogenized fecal samples using the QIAamp Fast DNA Stool Mini Kit (Qiagen, Hilden, Germany) as per the manufacturer's instructions, with minor modifications, i.e., two $0.05 \mathrm{M}$ phosphate buffer washes prior to the addition of InhibitEX (Qiagen) and a $1 \mathrm{~mm}$ zirconia/silica bead beating step $(\sim 250-350 \mathrm{mg} /$ tube, $4 \mathrm{~m} / \mathrm{s}$ for $1 \mathrm{~min} \times 3)$ before centrifugation of samples to pellet stool particles.
Purified DNA will be further processed for qPCR and $16 \mathrm{~S}$ rRNA gene amplicon sequencing.

For qPCR, template DNA for standard curves will be generated by spiking feces with a bacterial suspension consisting of lyophilized bacterial powder (Lallemand Health Solutions) in HyClone phosphate buffered saline. Total cell count of each bacterial suspension will be determined by flow cytometry. Feces will be spiked with a volume equivalent to $10^{9}$ bacteria, and then subjected to DNA extraction as described above.

Quantification of Lactobacilli, Bifidobacteria, and Bifidobacterium animalis sp. lactis will be performed by qPCR using the $\mathrm{ViiA}^{\text {ma }} 7$ Real-Time PCR System (Thermo Fisher Scientific). Standard curves will be generated by serially diluting DNA from spiked feces. DNA samples to be quantified will be diluted in molecular biology grade water prior to qPCR.

The qPCR reaction mixture will consist of the appropriate primers, $1 \mathrm{X} \mathrm{SYBR}^{\circ}$ Select Master Mix (Thermo Fisher Scientific), and diluted DNA. Standard curve samples will be run in duplicate and unknown samples will be tested in triplicate. Cycling conditions will consist of initial incubations, followed by 40 cycles of denaturation, annealing, and extension. A dissociation curve analysis $\left(60^{\circ} \mathrm{C}\right.$ to $\left.95^{\circ} \mathrm{C}\right)$ will also be performed to ensure amplification specificity of the primers.

Libraries for sequencing will be prepared according to Illumina's 16S Metagenomic Sequencing Library Preparation guidelines (Part \# 15044223 Rev. B), with the exception of using Qiagen HotStar MasterMix for the first PCR ('amplicon PCR') and halving reagent volumes for the second PCR ('index PCR'). As per Illumina's guidelines, template-specific primers will target the V3-V4 region of the 16S rRNA gene (PMCID: PMC3592464) [11]. Resulting sequence reads will be analyzed through the National Research Council's (Montreal, Canada) $16 \mathrm{~S}$ rRNA gene amplicon analysis pipeline, as previously described $[12,13]$. Reads will be QCed, paired-end assembled, and clustered at 97\% similarity. Taxonomic summaries and alpha (observed) and beta (weighted or unweighted UniFrac and Bray-Curtis distances) diversity metrics, statistical analysis, and taxonomic classifications will be computed using QIIME software [14] and downstream analyses will be performed with in-house Perl and R scripts at the National Research Council.

\section{Sample size determination}

For the power calculation, mean differences between the treatment group and the placebo group were obtained from Ishizuka et al. [15], who reported that, for constipated subjects, the mean frequency of bowel movements per week was 3.8 at baseline, 4.4 in the second week for those on placebo, and 5.1 in the second week for those on treatment. Data were simulated for each of the two 
experimental designs for 10,000 subjects assuming correlated Poisson random variables with mean weekly bowel movements as given above. Either the weekly totals as simulated or the averages of the weekly totals for each period (baseline, intervention) were used in subsequent analyses. For the simulations, all washouts were assumed to have the same mean as the placebo (4.4 bowel movements/week) and no temporal trend within each intervention or washout period was assumed. A correlation of 0.35 for the repeated observations on a subject was assumed. This value was obtained from previous studies involving measurement of weekly total bowel movements such as that of Baird et al. [16]. All power analyses were based on using a type I error rate of 0.05 and no correction for possible multiple comparisons. A cross-over study with five time periods, i.e., 4 weeks of baseline, 4 weeks on one treatment, 4 weeks of washout, 4 weeks on the alternative treatment, and 4 weeks posttreatment, was considered. Within each time period, the weekly totals to obtain an average total within each period for each subject were averaged. Hence, the value for any time period for a subject is the mean of the 4 weekly totals. Hypothesis testing included the F-tests in the ANOVA table for 'Period' and a $t$ test of the difference between the product and placebo. The recommended sample size for this model is 26 to determine a difference between treatments. If the weekly means and then the treatment differences by averaging the least squares means are tested after analysis, the same sample size is required, confirming the appropriate sample size of 26 . A washout of 4 weeks is expected to be sufficient to minimize any carryover effects. However, with a possibly high dropout rate of approximately $25 \%$ (due to the length of the study), a sample size of 36 is targeted. The targeted sample size is feasible as recruitment will be from a population that includes individuals participating in residential programming and those residing in the community at large.

\section{Statistical analysis}

For the primary outcome of stool frequency, data will be analyzed using a general linear mixed model with intervention, week, period, sequence, and the interactions of period, sequence and intervention with week (weekly data), as the main fixed effects. A random effect of subject will be included in the models. Pairwise tests and post-hoc analyses will be conducted using the Tukey-Kramer method. Kenward-Roger adjustments for the denominator degrees of freedom will be performed to adjust for bias in the covariance estimates for the random effect.

\section{Data management}

Data and files will be secured in locked cabinets and office space. Paper questionnaires will only include date and assigned participant number. Data that is originally captured as hardcopy/paper (e.g., questionnaires, etc.) will be transcribed to encrypted electronic files. Following the completion of the study, identifiers will be removed from all data.

\section{Discussion}

In this paper, we present a clinical trial design to evaluate the effects of the probiotic B. lactis B94 in adults with PWS. B. lactis B94 may increase stool frequency, decrease the percentage of slow transit stools, and improve GI symptoms. When consuming the probiotic, subjects may experience improved bowel habits.

This study has limitations. The sample size was determined based on a study of constipated adults as no known published research has explored B. lactis administration in PWS subjects. As PWS is a unique patient population, their response to $B$. lactis may differ from those without such a diagnosis. A differing response is possible due to potential disease-specific changes in GI function in individuals with PWS or dissimilarities in dietary intake as individuals with PWS are commonly prescribed energyrestricted diets [3], both of which may impact baseline microbiota and response to $B$. lactis. In addition, bowel habits of individuals with PWS residing in a residential home environment may differ from adults living independently. Furthermore, given the limited population to sample, we are recruiting individuals with PWS independent of constipation status and thus, non-constipated participants may differ in their response to $B$. lactis.

To our knowledge, this is the first randomized, controlled trial evaluating the effectiveness of a $B$. lactis strain on GI wellness in adults with PWS. The results of this study will provide information on the efficacy of $B$. lactis B94 in populations at risk for slow transit and constipation. In addition to pursing publication of the findings of this trial, the authors plan to communicate the results to the study participants and healthcare professionals involved in the care of individuals with PWS.

\section{Trial status}

The current protocol (IRB201701976, version 3) was approved December 4, 2017. Study recruitment began December 2017 and is expected to be completed by December 2018.

\section{Additional files}

Additional file 1: SPIRIT checklist. (PDF $169 \mathrm{~kb}$ )

Additional file 2: World Health Organization Trial Registration Data Set. (PDF $98 \mathrm{~kb}$ )

Abbreviations

BSFS: Bristol Stool Form Scale; CFU: Colony forming units; Gl: gastrointestinal; GSRS: Gastrointestinal Symptom Rating Scale; IP: investigational product; PI: principal investigator; PWS: Prader-Willi syndrome 


\section{Acknowledgements}

$Z Y$ is currently receiving a doctoral assistantship through the King Abdullah Scholarship Program sponsored by the Saudi Arabian Cultural Mission (SACM). This study will be carried out with in-kind support of the Food Science and Human Nutrition Department, University of Florida/Institute of Food and Agricultural Sciences (UF/IFAS).

\section{Funding}

This study is financially supported by Lallemand Health Solutions Inc. The sponsor of the trial is the University of Florida (W.J. Dahl, wdahl@ufl.edu). The funder has had no role in the study design, and will have no role in the collection, management, analysis, and interpretation of the primary outcome and the clinical secondary outcomes. The funder will be involved in the analysis and interpretation of the exploratory microbiota analysis. The University of Florida will retain access to the datasets and the results will be made available to the funder.

\section{Authors' contributions}

$Z Y, W J D$, and JLM contributed to the general idea. ZY, WJD, and JLM participated in the design of the study. ZY and WJD wrote the protocol and prepared the final version of this manuscript. ZY, WJD, and JLM will be responsible for recruitment and data collection. ZY and WJD, in consultation with a statistician, will be responsible for the data analyses of the primary and clinical secondary outcomes. MS, CM, and VN will be responsible for the analysis of the exploratory microbiome outcome. All authors have read and approved the final manuscript.

\section{Ethics approval and consent to participate}

This study was approved by the University of Florida's Institutional Review Board 1 (IRB-1) on 19 September 2017 protocol \#IRB201701976. Approval will be sought through the IRB-1 for any future revisions to the protocol and will be updated at clinicaltrials.gov. All subjects will be interviewed and provide written informed consent to participate in this study.

\section{Competing interests}

The authors ZA, WJD, and JLM declare that they have no competing interests. MS, CM, and VN are employed by Lallemand Health Solutions, the sponsor of this investigation.

\section{Publisher's Note}

Springer Nature remains neutral with regard to jurisdictional claims in published maps and institutional affiliations.

\section{Author details}

${ }^{1}$ Food Science and Human Nutrition Department, UF/IFAS, University of Florida, 359 FSHN Building Newell Drive, Gainesville, FL 32611, USA.

2Department of Pediatrics, College of Medicine, University of Florida, 2000 SW Archer Rd, Gainesville, FL 32608, USA. ${ }^{3}$ Lallemand Health Solutions, 6100 Royalmount, Montréal, QC H4P2R2, Canada.

Received: 19 October 2017 Accepted: 13 April 2018

Published online: 27 April 2018

\section{References}

1. Cassidy SB, Schwartz S, Miller JL, Driscoll DJ. Prader-Willi syndrome. Genet Med. 2012;14(1):10-26.

2. Lloret-Linares C, Faucher P, Coupaye M, Alili R, Green A, Basdevant A, et al. Comparison of body composition, basal metabolic rate and metabolic outcomes of adults with Prader Willi syndrome or lesional hypothalamic disease, with primary obesity. Int J Obes. 2013;37(9):1198-203.

3. Salehi P, Leavitt A, Beck AE, Chen ML, Roth CL. Obesity management in Prader-Willi syndrome. Pediatr Endocrinol Rev. 2015;12(3):297-307.

4. Kuhlmann L, Joensson IM, Froekjaer JB, Krogh K, Farhol S. A descriptive study of colorectal function in adults with Prader-Willi Syndrome: high prevalence of constipation. BMC Gastroenterol. 2014;14:63.

5. Miller JL, Lynn CH, Shuster J, Driscoll DJ. A reduced-energy intake, wellbalanced diet improves weight control in children with Prader-Willi syndrome. J Hum Nutr Diet. 2013;26(1):2-9.

6. Joint FAO/WHO Working Group. Guidelines for the Evaluation of Probiotics in Food. Geneva and Rome: World Health Organization and Food and Agriculture Organization; 2002.
7. Dimidi E, Christodoulides S, Fragkos KC, Scott SM, Whelan K. The effect of probiotics on functional constipation in adults: a systematic review and metaanalysis of randomized controlled trials. Am J Clin Nutr. 2014;100(4):1075-84.

8. Lewis SJ, Heaton W. Stool form scale as a useful guide to intestinal transit time. Scand J Gastroenterol. 1997;32(9):920-4.

9. Svedlund J, Sjodin I, Dotevall G. GSRS-a clinical rating scale for gastrointestinal symptoms in patients with irritable bowel syndrome and peptic ulcer disease. Dig Dis Sci. 1988;33(2):129-34.

10. Kulich KR, Madisch A, Pacini F, Pique JM, Regula J, Van Rensburg CJ, et al. Reliability and validity of the Gastrointestinal Symptom Rating Scale (GSRS) and Quality of Life in Reflux and Dyspepsia (QOLRAD) questionnaire in dyspepsia: a six-country study. Health Qual Life Outcomes. 2008;6:12.

11. Klindworth A, Pruesse E, Schweer T, Peplies J, Quast C, Horn M, et al. Evaluation of general 165 ribosomal RNA gene PCR primers for classical and next-generation sequencing-based diversity studies. Nucleic Acids Res. 2013;41(1):e1.

12. Yergeau E, Bell TH, Champagne J, Maynard C, Tardif S, Tremblay J, Greer CW. Transplanting soil microbiomes leads to lasting effects on willow growth, but not on the Rhizosphere microbiome. Front Microbiol. 2015;6:1436.

13. Tremblay J, Singh K, Fern A, Kirton ES, He S, Woyke T, et al. Primer and platform effects on 16S rRNA tag sequencing. Front Microbiol. 2015;6:771.

14. Caporaso JG, Kuczynski J, Stombaugh J, Bittinger K, Bushman FD, Costello EK, et al. QIIME allows analysis of high-throughput community sequencing data. Nat Methods. 2010;7(5):335-6.

15. Ishizuka A, Tomizuka K, Aoki R, Nishijima T, Saito Y, Inoue R, et al. Effects of administration of Bifidobacterium animalis subsp. lactis GCL2505 on defecation frequency and bifidobacterial microbiota composition in humans. J Biosci Bioeng. 2012;113(5):587-91.

16. Baird IM, Walters RL, Davies PS, Hill MJ, Drasar BS, Southgate DA. The effects of two dietary fiber supplements on gastrointestinal transit, stool weight and frequency, and bacterial flora, and fecal bile acids in normal subjects. Metabolism. 1977;26(2):117-28.

\section{Ready to submit your research? Choose BMC and benefit from:}

- fast, convenient online submission

- thorough peer review by experienced researchers in your field

- rapid publication on acceptance

- support for research data, including large and complex data types

- gold Open Access which fosters wider collaboration and increased citations

- maximum visibility for your research: over $100 \mathrm{M}$ website views per year

At BMC, research is always in progress.

Learn more biomedcentral.com/submissions 